\title{
Changes in the organization of the genome during the mammalian cell cycle
}

\author{
Luca Giorgetti ${ }^{1 *}$, Nicolas Servant ${ }^{2}$ and Edith Heard ${ }^{1}$
}

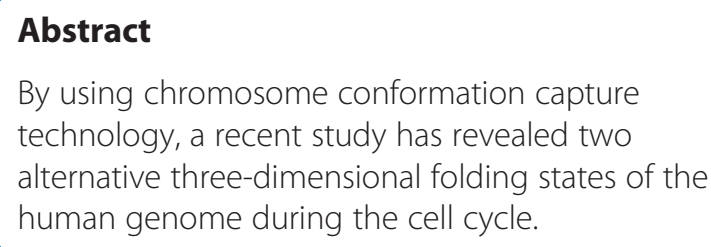

By using chromosome conformation capture technology, a recent study has revealed two alternative three-dimensional folding states of the human genome during the cell cycle.

\section{The packaging conundrum at ever-higher levels of scrutiny}

Understanding the spatial organization of chromosomes represents a major quest, not only for our comprehension of how a length of $2 \mathrm{~m}$ of DNA can be packaged into a nucleus of just a few microns, but also because the physical interactions occurring within and between chromosomes are thought to play an important role in gene regulation, DNA replication and genome stability. Over recent years, the development of chromosome conformation capture (3C)-based techniques, together with the emergence of next-generation sequencing, have changed our view of nuclear organization. High-throughput conformation capture techniques have been developed to study the physical interactions of the chromatin fiber with or within genomic regions spanning from a few megabases ( $5 \mathrm{C}$ technique) to a whole-genome scale (4C and $\mathrm{Hi}-\mathrm{C}$ techniques). In 2009, Eric Lander, Job Dekker and colleagues [1] described the first human chromosomal architecture at a resolution of $1 \mathrm{Mb}$. Then, four years later, owing to the rapid evolution of sequencing capabilities, Bing Ren and colleagues [2] published a high-resolution map of the 'chromatin interactome' in human fibroblasts at a resolution of 5 to $10 \mathrm{~kb}$.

The picture that emerges from this blossoming area of research is that metazoan chromosomes are characterized by a nested hierarchy of structural layers (Figure 1, left panel). Within each chromosome territory, compartments originally termed ' $\mathrm{A}$ ' and ' $\mathrm{B}$ ', each of several megabases, tend to associate within each single chromosome,

\footnotetext{
* Correspondence: luca.giorgetti@curie.fr

${ }^{1}$ Institut Curie, CNRS UMR3215, INSERM U934 26 rue d'Ulm, Paris F-75248,

France

Full list of author information is available at the end of the article
}

reflecting the preferential colocalization of active, generich regions and their segregation from gene-poor, inactive regions [1]. More recently, a further submegabase scale of genome partitioning into topologically associating domains (TADs) has been reported [3-5]. Sequences within TADs tend to interact more frequently than with any other surrounding region of the genome. These domains, spanning a few hundred kilobases, are stable across different cell types, suggesting that they are an inherent property of mammalian genomes. Furthermore, within TADs, a network of cell-type-specific physical interactions between potential regulatory sequences has been shown to take place [6]. Consistent with this, a recent study using high-resolution $\mathrm{Hi}-\mathrm{C}$ showed that enhancer-promoter contacts almost always occur within TADs [2].

Despite the pace at which new details of mammalian chromosome organization are accumulating, many fundamental questions remain unanswered. For example, what is the cell-to-cell variability in the structures that give rise to compartments, TADs and promoter-enhancer interactions? How important is each of those structural layers for the regulation of transcription? What are the molecular mechanisms that determine the appearance of TADs and compartments? When are these layers established during development? Are these different levels of chromosome organization present throughout the cell cycle, or are they established at a particular phase? Finally, what is the structure of mitotic chromosomes? Are the hierarchical layers of folding maintained during mitosis, or do chromosomes acquire a different organization, as suggested by light and electron microscopy? A recent exciting study has shed light on these last questions [7].

\section{Compartments and TADs are erased during mitosis}

Although DNA fluorescence in situ hybridization (FISH) coupled to super-resolution microscopy has previously 


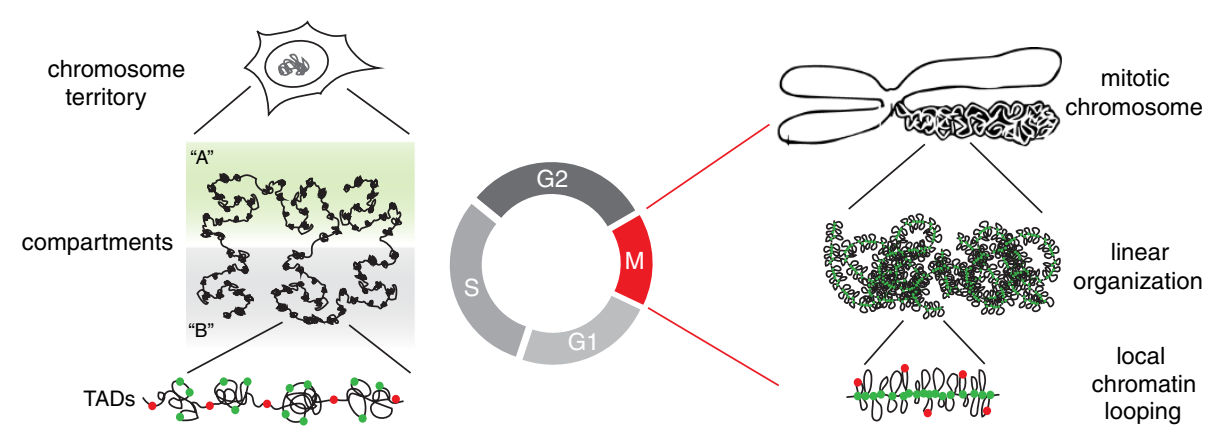

- structural bookmarking factor (?)

Figure 1 Cell-cycle dependence of chromosome structure. Left: high-resolution chromosome-conformation capture technologies have revealed that each mammalian chromosome territory can be decomposed into multi-megabase compartments where preferential interactions occur between active gene-rich and inactive gene-poor regions of the chromosome. Compartments can be further partitioned at the submegabase scale into topological associating domains (TADs) spanning a few hundred kilobases each. Dekker and colleagues [7] show that compartments and TADs are stable across the cell cycle from G1 to late S phase. Right: in metaphase, the compartment and TAD structures disappear and are replaced by a cylindrically condensed structure of linearly arranged short-range loops [7], which occur on a shorter genomic scale (80 kb on average) than TADs. It is hypothesized that the persistence of bookmarking factors on the mitotic chromosome might facilitate the rapid reemergence of compartments and TADs upon exit of the cells from mitosis.

been used in murine cells to investigate the presence of TADs within the X-inactivation center during the cell cycle from G1/S phase to mitotic prophase [4], a detailed molecular analysis of chromatin interactions throughout the cell cycle had not, until recently, been reported. Dekker and colleagues [7] now report the results of performing carbon-copy chromosome conformation capture (5C) and $\mathrm{Hi}-\mathrm{C}$ experiments in synchronized human HeLa cells at different phases of the cell cycle. Using 5C technology spanning the whole of chromosome 21 at a resolution of $250 \mathrm{~kb}$, the authors first studied the long-range chromatin interactions of early G1-, mid-G1-, S- and M-phase cells. Interaction maps of these different stages showed that the mitotic interaction pattern differs dramatically from that at all other stages, thus revealing two distinct chromosome folding states during the cell cycle. Using $\mathrm{Hi}-\mathrm{C}$ (at a resolution of $40 \mathrm{~kb}$ ) on mitotic and mid-G1-stage cells [7], the investigators further found that both compartments [1] and TADs [3,4] appear to be absent throughout the entire genome in metaphase, becoming detectable only in early G1 phase and remaining unchanged throughout interphase. These observations on mitotic chromosomes were repeated and validated in another cell line and in primary human fibroblasts. Importantly, the authors showed that, apart from metaphase, compartments and TADs are not restricted to any specific cell cycle phase and they are easily detected in early G1, thus ruling out the possibility that the patterns observed in $5 \mathrm{C}$ or $\mathrm{Hi}-\mathrm{C}$ could be due to a superposition of alternative cell-cycle phase-specific conformations.

The finding that TADs and compartments apparently disappear during metaphase, and reappear in early G1, raises the questions of why they are lost and also what mechanism ensures their prompt re-establishment upon exit from mitosis. It is known that certain transcription factors and histone modifications or associated factors can act as 'bookmarking' factors on mitotic chromosomes [8] by remaining bound to the condensed chromatin polymer in order to ensure the propagation of transcriptional states to daughter cells. It is therefore tempting to speculate that structural bookmarking factors that propagate organizational information to daughter cells might similarly exist. Although the molecular bases of compartments and TADs remain elusive, accumulating evidence suggests that long-range interactions between genomic sites bound by the proteins CTCF and cohesin contribute to the organization of chromatin architecture at the TAD scale, either by associating with TAD boundaries [3] or by supervising a network of long-range interactions inside single TADs that might stabilize the structure within TADs [6]. Interestingly, both CTCF and cohesin have been shown to associate with mitotic chromosomes [8], suggesting that mutual interactions between CTCF and/or cohesin binding sites might readily occur upon exit from mitosis, thus enabling TADs to be established immediately at the beginning of G1 phase. Other factors that might remain bound during mitosis to the positions of TAD or 
compartment boundaries include Polycomb group proteins, which have been shown to significantly overlap with TAD boundaries [5] on mitotic chromosomes in Drosophila [9]

\section{A polymer view of a mitotic chromosome}

The apparent loss of partitioning into TADs and compartments observed in mitotic chromosomes by Dekker and colleagues [7] suggests that they contain unusually few structural details compared with those of their interphase counterparts. However, the authors cleverly exploited the quantitative information present in the Hi$\mathrm{C}$ mitotic chromosome data to build a structural model. The foundation of their approach is that $\mathrm{Hi}-\mathrm{C}$ or $5 \mathrm{C}$ interaction maps represent the frequency at which every pair of genomic loci along the chromatin polymer encounter each other, averaged over millions of cells. It is therefore possible to use models from polymer physics to simulate virtual $\mathrm{Hi}-\mathrm{C}$ (or $5 \mathrm{C}$ ) data and compare them with data from experiments. Using such an approach, the thermodynamic ensemble of configurations of a model chromosome can be generated by computer simulations, and a contact map is retrieved by averaging the contacts of each pair of loci over all simulated polymer conformations, allowing a straightforward comparison with experimental data.

In the case of mitotic chromosome data, the $\mathrm{Hi}-\mathrm{C}$ or $5 \mathrm{C}$ contact maps are homogeneous, with no sign of the regular patterning due to TADs and compartments that characterize the interphase contact maps [7]. Dekker and colleagues also noted that the contact probability between two loci gradually decreases with increasing genomic distance (albeit significantly more slowly than on interphase chromosomes), and then suddenly falls off to zero at approximately $10 \mathrm{Mb}$. This unusual behavior cannot be explained by any simple polymer models. Building on the well-established experimental evidence describing the quantitative properties of mitotic chromosomes (such as cylindrical symmetry, linear organization of chromatids and chromatin packing density), the authors thus set out to build various alternative models for the chromatin organization within mitotic chromosomes, simulated all of them and tested each model's predictions. In order to reproduce the unexpected falloff in contact probabilities for loci that are separated by $>10 \mathrm{Mb}$, they had to impose that the chromatin fiber is linearly organized, so that genomic loci belonging to distal parts of the chromosome (that is, separated by $>10 \mathrm{Mb}$ ) cannot be brought into close spatial proximity. Furthermore, they had to impose that the chromatin fiber is arranged in an array of consecutive loops, each spanning approximately $80 \mathrm{~kb}$, in order for their simulations to account correctly for the gentle decrease in contact frequencies in the genomic length range between 0 and $10 \mathrm{Mb}$. Thus, amongst all possible models tested, it was this combination of linear organization and consecutive looping that best accounted for the behavior of the contact probability of mitotic chromosomes over all genomic length scales. Interestingly, the authors further discovered that they could simultaneously reproduce the scaling of contact probabilities and the homogeneity of the mitotic contact maps only when they allowed the size of the loops to be different (and stochastic) in each of the polymer configurations.

\section{Future perspectives}

The study by Dekker and colleagues represents a powerful combination of biochemical investigation and polymer modeling [7]. The prowess of such an approach is that it can provide insights into structure that would not have been obtained without modeling. The picture that emerges, thanks to their study, is that of a mitotic chromosome composed of consecutive loops of chromatin, the size of which is on average $80 \mathrm{~kb}$, although this varies from cell to cell, which are arranged in a linear fashion eventually resulting in an effective cylindrical chromosomal volume (Figure 1, right panel). This picture is both qualitatively and quantitatively in agreement with previously proposed 'loops-on-a-scaffold' models of mitotic chromosomes based on microscopy and biochemical assays.

Although the molecular mechanisms that could mediate this pervasive local looping remain elusive, the model predicts that cell-to-cell variability of looping events has a sizeable effect in organizing the structure of mitotic chromatin. Indeed, the potential importance of fluctuations in interphase chromosome structure was also recently revealed by an adaptation of the $\mathrm{Hi}-\mathrm{C}$ technique to single cells [10]. In the future, this type of single-cell approach, together with physical modeling and quantitative analysis, could allow the exploration of chromosome structure in situations where cell-to-cell variability might be crucial, such as during early development.

\section{Abbreviations \\ 3C: Chromosome conformation capture; 4C: Circularized chromosome conformation capture; 5C: Carbon-copy chromosome conformation capture; Hi-C: High-throughput conformation capture; TAD: Topologically associating domain.}

\section{Competing interests}

The authors declare that they have no competing interests.

\section{Acknowledgements}

LG was supported by an EMBO Fellowship (ALTF 1559-2011); work in the lab of EH is supported by the "Ligue Nationale contre le cancer", the EpiGeneSys FP7 257082 Network of Excellence, ERC Advanced Investigator award 250367 and EU FP7 MODHEP EU grant no. 259743. 


\section{Author details}

'Institut Curie, CNRS UMR3215, INSERM U934 26 rue d'Ulm, Paris F-75248,

France. ${ }^{2}$ Institut Curie, INSERM U900, Bioinformatics and Computational

Systems Biology of Cancer, 26 rue d'Ulm, Paris F-75248, France.

Published: 24 December 2013

\section{References}

1. Lieberman-Aiden E, Berkum NL V, Williams L, Imakaev M, Ragoczy T, Telling A, Amit I, Lajoie BR, Sabo PJ, Dorschner MO, Sandstrom R, Bernstein B, Bender MA, Groudine M, Gnirke A, Stamatoyannopoulos J, Mirny LA, Lander ES, Dekker J: Comprehensive mapping of long-range interactions reveals folding principles of the human genome. Science 2009, 326:289-293.

2. Jin F, Li Y, Dixon JR, Selvaraj S, Ye Z, Lee AY, Yen C-A, Schmitt AD, Espinoza $C A$, Ren $B: A$ high-resolution map of the three-dimensional chromatin interactome in human cells. Nature 2013, 503:290-294.

3. Dixon JR, Selvaraj S, Yue F, Kim A, Li Y, Shen Y, Hu M, Liu JS, Ren B: Topological domains in mammalian genomes identified by analysis of chromatin interactions. Nature 2012, 485:376-380.

4. Nora EP, Lajoie BR, Schulz EG, Giorgetti L, Okamoto I, Servant N, Piolot T, van Berkum NL, Meisig J, Sedat J, Gribnau J, Barillot E, Blüthgen N, Dekker J, Heard E: Spatial partitioning of the regulatory landscape of the Xinactivation centre. Nature 2012, 485:381-385.

5. Sexton T, Yaffe E, Kenigsberg E, Bantignies F, Leblanc B, Hoichman M, Parrinello H, Tanay A, Cavalli G: Three-dimensional folding and functional organization principles of the Drosophila genome. Cell 2012, 148:458-472.

6. Phillips-Cremins JE, Sauria MEG, Sanyal A, Gerasimova TI, Lajoie BR, Bell JSK, Ong C-T, Hookway TA, Guo C, Sun Y, Bland MJ, Wagstaff W, Dalton S, McDevitt TC, Sen R, Dekker J, Taylor J, Corces VG: Architectural protein subclasses shape 3D organization of genomes during lineage commitment. Cell 2013, 153:1281-1295.

7. Naumova N, Imakaev M, Fudenberg G, Zhan Y, Lajoie BR, Mirny LA, Dekker J: Organization of the mitotic chromosome. Science 2013, 342:948-953.

8. Kadauke S, Blobel GA: Mitotic bookmarking by transcription factors. Epigenetics Chromatin 2013, 6:6.

9. Follmer NE, Wani AH, Francis NJ: A Polycomb group protein is retained at specific sites on chromatin in mitosis. PLOS Genet 2012, 8:e1003135.

10. Nagano T, Lubling Y, Stevens TJ, Schoenfelder S, Yaffe E, Dean W, Laue ED, Tanay A, Fraser P: Single-cell Hi-C reveals cell-to-cell variability in chromosome structure. Nature 2013, 502:59-64.

doi:10.1186/gb4147

Cite this article as: Giorgetti et al:: Changes in the organization of the genome during the mammalian cell cycle. Genome Biology 2013 14:142. 